\title{
3D Model-Based Approach to Lung Registration and Prediction of Respiratory Cardiac Motion
}

\author{
Mikhail G. Danilouchkine^, Jos J.M. Westenberg, Hans C. van Assen, \\ Johan H.C. van Reiber, and Boudewijn P.F. Lelieveldt \\ Division of Image Processing, Dept. Radiology, Leiden University Medical Center, \\ Leiden, The Netherlands
}

\begin{abstract}
This paper presents a new approach for lung registration and cardiac motion prediction, based on a 3D geometric model of the left lung. Feature points, describing a shape of this anatomical object, are automatically extracted from acquired tomographic images. The "goodness-of-fit" measure is assessed at each step in the iterative scheme until spatial alignment between the model and subject's specific data is achieved. We applied the proposed methods to register the 3D lung surfaces of 5 healthy volunteers of thoracic MRI acquired in different respiratory phases. We also utilized this approach to predict the spatial displacement of the human heart due to respiration. The obtained results demonstrate a promising registration performance.
\end{abstract}

\section{Introduction}

Over the past decennium image registration has been receiving a significant amount of attention from the medical image processing community. Registration methods aim at establishing a spatial alignment between the tomographic images that were taken at different time points or under different conditions or with different imaging modalities. A vast number of different registration algorithms have been proposed and exhaustively reviewed in literature [1. Registration algorithms can be split into the following broad categories: intensity- 223] and feature-based 45. methods or a hybrid of both approaches 6].

The intensity-based methods provide the most accurate way for spatial alignment between two image sets. A registration is performed by transforming one image set with respect to the other and optimizing a measure based on pairs of overlapping voxels. Different similarity measures (i.e. normalized cross correlation, mutual information of pixel intensities etc.) have been adopted to govern the optimization procedure. In spite of the fact that the intensity-based registration algorithms are optimal in terms of accuracy, they remain computationally expensive. The feature-based methods offer a better alternative with respect to numerical efficiency. Commonly two surfaces or point sets, describing the shape

\footnotetext{
* Partially funded by the Dutch Foundation for Technical Sciences (STW LPG 5651). M.Danilouchkine is now at the Dept. Cardiology, Erasmus Medical Center, Rotterdam, the Netherlands.
} 
of the same anatomical objects, are aligned with each other, based on a mean squared distance metric. The feature-based registration methods are extremely sensitive to initial mutual alignment of the feature sets.

In this paper, we propose a new model-based registration approach, that combines the best features of the registration algorithms of both categories. We strive to achieve the accuracy comparable with one of the intensity-based registration methods without sacrificing the computational efficiency. Moreover, the model-based approach is much less sensitive to initial misalignment of the feature points with the geometric model.

In this work we adopted the model-driven segmentation method for thoracic MRI images and automated recognition of the organs with the profound airtissue boundaries proposed in [7. A new concept of multi-resolution registration is introduced. It significantly improves the computational efficiency and convergence to the globally optimal solution. The energy minimization functional, reflecting a "goodness-of-fit" measure, was adapted to meet the new requirements. This approach is applied to registration of left lung MRI images in extreme respiratory states and to predict the cardiac motion due to respiration.

\section{Methods}

The components of the model-based registration framework and their interconnections are shown in Fig 1. The basic input data for the registration process are the geometric model of an anatomical organ and the representative set of the feature points describing the same organ. Registration is treated as an optimization problem aimed at finding the spatial mapping that aligns the feature points with the geometric model. The transform component represents the spatial mapping of the features points to the model space. Metric provides a "goodness-of-fit" measure of how well the feature points are brought into alignment with the

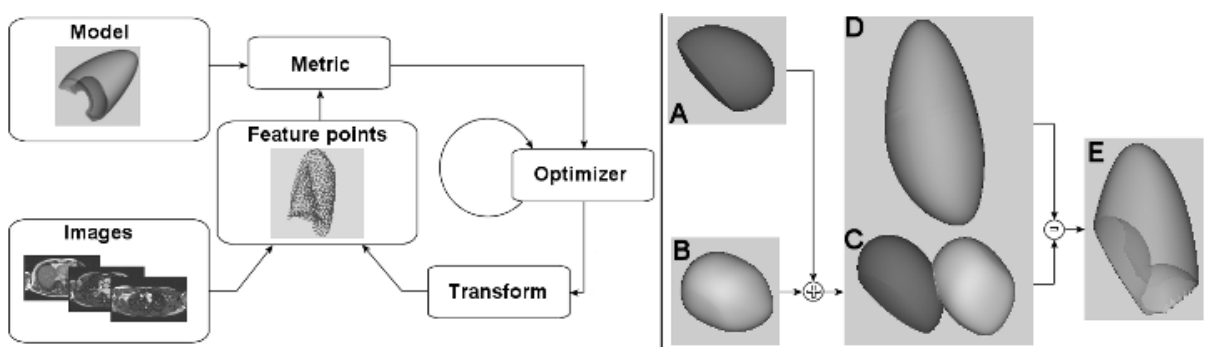

Fig. 1. The main components of the model-based registration framework (left panel). The right panel depicts the 3D geometric model, consisting of the heart (A), spleen (B) and left lung (D) primitives. Those primitives are combined together by means of boolean operators, e.g. union-operator for the heart-spleen composite $(\mathrm{C})$ and difference-operator for the final model (E), describing the complex shape of the left lung. 
model. This measure is the quantitative criterion to be optimized by the optimizer over the search space defined by the transformation parameters.

\subsection{D Geometric Model of Left Lung}

Among the vast variety of different modeling paradigms, Implicit Solid Modeling (ISM) 8] allows to describe the shape of an anatomical object in terms of an algebraic implicit function of the spatial coordinates $F(x, y, z)$. The set of points where the function has a predefined cutoff value $c$ describes the surface of the modeled anatomical object. Its interior and exterior are associated with the points where $F(x, y, z)<c$ and $F(x, y, z)>c$, respectively. Different F's can be deduced to describe the analytical template, called primitive, of different anatomical objects. Primitives are organized in a tree-like structure to describe intermediate composites and the final model. The primitives form the leaves of the tree, while the nodes are boolean operators such as differences, unions, etc. The geometric properties of this model are thus expressed in the primitives, while the topological characteristics are stored in the relational tree. Fig. 1 shows the $3 \mathrm{D}$ geometric model of the left lung constructed by means of ISM.

\subsection{Feature Points}

The feature points, describing the shape of the modeled anatomical organ (e. g. left lung), can be extracted automatically from the tomographic images of the examined subject. A cumulative pixel-intensity histogram of scout images typically has a bimodal distribution: one peak corresponds to the pixels with low attenuation or air, contained in the lungs, and the other peak is attributed to tissue or thoracic organs. Expectation-Maximization [9] histogram-based clustering can be utilized to determine the brightness values for air and tissue classes. By fitting a two-component mixture of Gaussian distributions and assigning the class labels according to a maximum a-posteriori criterion, the final segmentation of the scout images can be obtained. A set of tuples $\left(x_{i}, g_{i}\right)$ constitutes the automatically detected feature points, where $x_{i}$ is a vector of the spatial coordinates of the air-tissue boundaries and $g_{i}$ is the image gradient at the point $x_{i}$.

\subsection{Metric and Optimizer}

A metric characterizes the fit between the model and feature points. It is defined via the boundary distance field, which was first introduced in [7]. This field represents a mapping $b_{\Delta}: R^{3} \rightarrow R$ that computes a value for each point according to the following formula:

$$
b_{\Delta}\left(x_{i}\right)= \begin{cases}\left|d\left(x_{i}\right)\right| / \Delta, & \left|d\left(x_{i}\right)\right|<\Delta \\ 1, & \left|d\left(x_{i}\right)\right|>\Delta .\end{cases}
$$

where $d\left(x_{i}\right) \approx\left(F\left(x_{i}\right)-c\right) /\left\|\bar{\nabla} F\left(x_{i}\right)\right\|$ is the orthogonal distance from the feature point $x_{i}$ to the surface of the model; $\Delta$ is the effective range of the boundary distance field. The boundary distance value is equal to 0 for the points lying on 
the model surface, and exhibits approximately linear behavior in the vicinity of the surfaces as the distance to the model surface increases. Outside the effective range, this value is equal to 1 .

The energy minimization function to be optimized is given by the formula:

$$
\begin{aligned}
& E\left(x_{i}, g_{i}\right)=\sum_{i=1}^{N} w\left(x_{i}, g_{i}\right) b_{\Delta}^{2}\left(x_{i}\right)+\rho \cdot \frac{N-\tilde{N}}{N}, \text { where } \\
& w\left(x_{i}, g_{i}\right)=\exp \left\{\alpha\left(1-\arccos \frac{\bar{\nabla} F\left(x_{i}\right) \cdot g_{i}}{\left\|\bar{\nabla} F\left(x_{i}\right)\right\| \cdot\left\|g_{i}\right\|}\right)\right\}
\end{aligned}
$$

The first term in Eq. 2 is a weighted sum of squares of the boundary distance values and quantifies proximity of the feature points to the model. The weighting factor $w$ assures the topological alignment of the feature points. It attains the minimum, when the image gradient lies along the direction of the model gradient, and maximum, when those vectors point in the opposite directions. The second term in Eq. 2 is a penalty factor proportional to the relative number of points lying outside the effective range of the distance field $(N$ - the total number of points and $\tilde{N}$ - the number of points inside the boundary distance field).

The energy minimization function can be optimized by introducing variations in the spatial positioning, scaling and rotation of the feature points. Since the minimization function is the sum of squares, a numerically stable method (e.g. Levenberg-Marquardt [10]) is used in the optimization procedure.

\subsection{Multi-resolution Optimization}

Multi-resolution approaches are usually employed to improve the computational efficiency and robustness of the registration algorithms. Initially the optimization

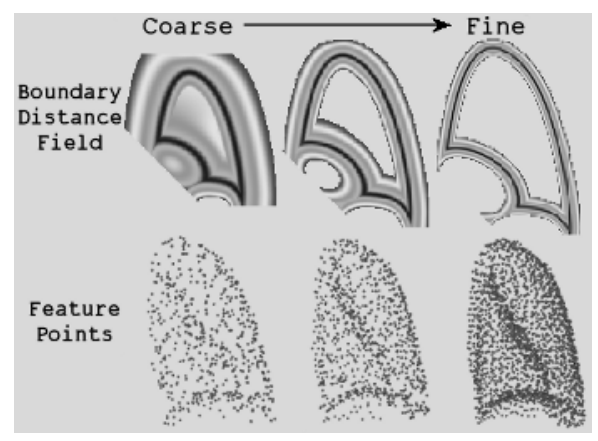

Fig. 2. Multi-resolution approach employed in the model-based registration. The varying effective range of the boundary scalar field (top row) improves robustness of the optimization procedure (boundary distance values are color coded with blue (dark) corresponding to 0 and yellow (light) - to 1), while the variable sparsity factor applied to the feature points (bottom row) increases the computational efficiency. 
procedure is solved at the coarsest level. The obtained results are used as a starting guess for finding a solution at the next finer level. The whole procedure iterates until the finest level is reached.

To improve the robustness of the model-based registration, the effective range of the boundary distance field depends on the resolution scale (Fig 2). At the coarsest level, this range is made large. The optimization procedure is primarily governed by the first term of the energy minimization function Eq. 2. The results obtained at a coarser level are subsequently propagated to the next finer level with reduced range of the boundary distance field. At the finer levels both terms in Eq. 2 are equally important. Due to the narrowing effective range, small variations in the spatial positioning, scaling and rotation of the feature points may result in some points moving outside the boundary scalar field, introducing irregularities in the energy minimization function. However, such variations are penalized by the second term, locking the feature points in the vicinity of the global solution and increasing the robustness of the optimization procedure.

The computational efficiency of the model-based registration algorithm is mainly determined by its numerical complexity. The latter can be roughly estimated as $O(M N)$, where $N$ is the number of the feature points and $M$ is the number of primitives constituting the geometric model. While the number of the primitives remains constant, the number of the feature points used at the different resolution scales is made variable. At a coarser level, the optimization procedure is applied to a sparser subset of the extracted feature points, yielding fewer computational costs. At the finer levels the number of the feature points is gradually increased, providing more accurate description of the object of interest and better mutual alignment with the model.

\section{Results}

In-vivo scout images were obtained from 5 healthy volunteers using a Philips Gyroscan Intera 1.5T MRI scanner. A balanced-FFE protocol (TR $2.32 \mathrm{~ms}$; TE $1.16 \mathrm{~ms}$; flip angle $55^{\circ}$ ) with prospective VCG was utilized to acquire 60 thoracic images in three standard projections (20 transversal, 20 sagittal, 20 coronal). Two sets of scout images were obtained at full inspiration and expiration for each subject. All volunteers were instructed to withhold from breathing during acquisition. The field of view and slice thickness were equal to $450 \mathrm{~mm}$ and $8 \mathrm{~mm}$, respectively. The reconstruction matrix of $256 \times 256$ was used, yielding the effective in-plane resolution of $1.75 \mathrm{~mm} /$ pixel.

In the first experiment, the model-based registration method was used to identify the anatomically homologous cross-sections of the left lung in extreme respiratory states. The geometric model of this anatomical organ provided the common reference frame for registration. Two sets of the feature points, describing the shape of the left lung of the examined person at full inspiration and expiration, were automatically extracted from the acquired scout images. Both sets were aligned with the model, resulting in two sets of the transformation parameters. A stack of parallel, equidistantly spaced intersecting planes, span- 


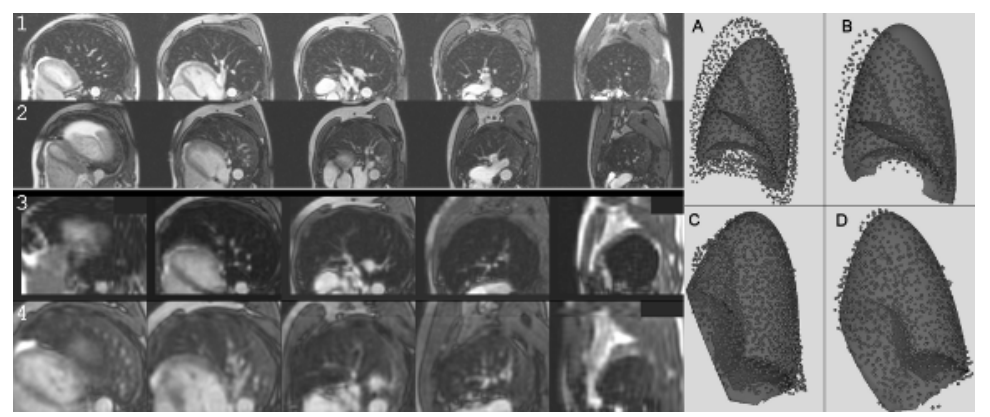

Fig. 3. The results of model-based registration. The spatially homologous cross-sections of the left lung (columns of the first two rows to the left), acquired at full inspiration (1st row) and expiration (2nd rows) show the effect of respiration. The morphologically homologous cross-sections (columns of last two rows to the left), reconstructed after registration, exhibit the anatomical coherence of the left lung appearance. Initial misalignment of the feature points at full inspiration (A) and expiration (B) does not affect the final registration results (C,D).

ning the whole extent of the geometric model in the axial direction, was used to identify the morphologically homologous cross-sections in in-vivo imaging data. The geometric parameters of those intersecting planes were projected back from the model into imaging domain by applying the inverse transformation, obtained during registration. The morphologically homologous cross-sections, reconstructed after registration, exhibit the anatomical coherence of the left lung in different slices and are shown in Fig. 3. Before registration, the lung size is generally smaller at full expiration in all slices. Moreover, the cross-section with the liver in the most inferior slice is only visible at full expiration. After registration, the size of the lung is approximately the same in all cross-sections.

Table 1. The results of the quantitative comparison of model- and intensity-based registration approaches (two first columns) using the normalized cross-correlation metric. The assessment of respiratory motion of the heart are summarized in last three columns. The levels of statistical insignificance were found for ${ }^{*}=0.54$ and for ${ }^{* *}=0.41$.

\begin{tabular}{rcc|ccc}
\hline \hline & \multicolumn{3}{|c|}{ NCC* $^{*}$} & \multicolumn{3}{c}{ Heart Displacement (mm) } \\
& Intensity-based & Model-based & Actual & Predicted & Abs.Diff. \\
\hline Normal 1 & 0.70 & 0.76 & 32.76 & 29.01 & 3.75 \\
Normal 2 & 0.92 & 0.79 & 26.66 & 30.62 & 3.96 \\
Normal 3 & 0.84 & 0.82 & 22.20 & 26.95 & 4.75 \\
Normal 4 & 0.73 & 0.72 & 32.35 & 28.37 & 3.98 \\
Normal 5 & 0.77 & 0.73 & 28.86 & 32.55 & 3.68 \\
\hline Mean & 0.79 & 0.76 & 28.57 & 29.50 & 4.03 \\
StdDev & 0.09 & 0.04 & 4.36 & 2.16 & 0.42 \\
\hline \hline
\end{tabular}



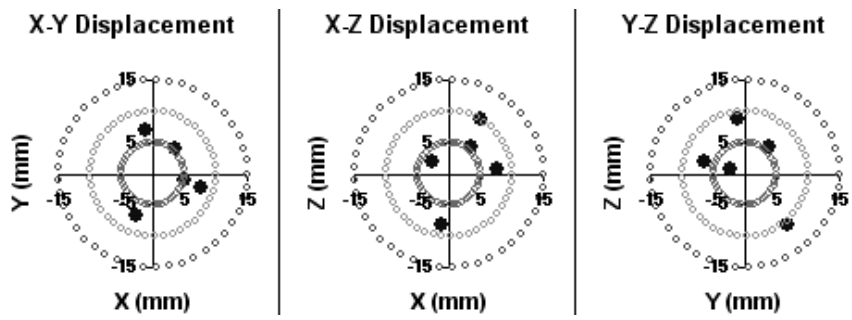

Fig. 4. Difference between actual and predicted heart displacement in projection onto the coordinate planes. The errors are of the same order of magnitude in all projections.

The accuracy of model-based registration was evaluated using the normalized cross-correlation (NCC). The correlation coefficient, normalized by the square root of the autocorrelation, was used to assess the similarity between the morphologically homologous stacks of the left lung at full expiration and inspiration. The NCC results for all subjects as well as the mean and standard deviation are presented in the second column of Tab. 1. For quantitative comparison, the intensity-based registration method was implemented using the Insight Toolkit (ITK) [1]. Prior to registration, the Volume-Of-Interest (VOI) containing the left lung was manually outlined for all subjects and two respiratory states. The affine image registration technique, based on normalized mutual information as a voxel similarity measure and the multi-resolution matching strategy, was employed to align the VOI's at full expiration and inspiration. The quality of intensity-based registration was finally measured using the NCC metric and is shown in the first column of Tab. 1

In our second experiment, we numerically assessed the displacement of the heart due to respiration. The actual and predicted displacements of the heart were quantitatively compared. The actual displacement was computed by manually delineating the left ventricular (LV) blood pool in all visible cross-sections of the chamber in the scout images, by estimation of the LV center by calculation of the center-of-gravity of the boundary points, and by quantifying the distance between these centers at full expiration and inspiration. The predicted displacement was estimated by back-projection of the center of the LV, comprising the model primitive, from the model to imaging domain using two inverse transformations, obtained during the registration in full exhalation and inhalation. The actual and predicted displacements of the heart along with the absolute difference are shown in last three columns of Tab. 1 and Fig. 4.

\section{Discussion and Conclusions}

The results show that this new algorithm is a promising tool for left lung registration. The model-based registration with the multi-resolution matching strategy is a fast way of establishing alignment of the left lung in different respiratory states and gives comparable accuracy as the intensity-based registration. The 
algorithm also produces plausible matches while depending less on correct initial alignment between the feature points and model (Fig. 3). Computational costs for model-based registration amount to 10-12 sec. per match against 60$75 \mathrm{sec}$. for the intensity-based registration method on a Pentium $2.8 \mathrm{GHz}$ with $1024 \mathrm{MB}$ memory capacity. In particular, the model-based registration is also suitable for the quantitative analysis of respiratory motion of the heart. Our findings are in excellent agreement with those of McLeish [12, obtained with the intensity-based rigid registration of the lungs in extreme respiratory phases.

The proposed model-based registration method was based on two assumptions. Firstly, ISM effectively but coarsely allows to represent the shape of anatomical organs with moderately complex shapes such as lungs and is less suitable to represent highly complex shapes such as cortical sulci or brain ventricles. Secondly, there is a single affine transformation that matches the set of feature points against the model. Although not strictly true, this assumption provides a global but accurate conformal mapping between lungs in extreme respiratory states. That mapping can be beneficial in establishing preliminary correspondence between the major anatomical landmarks of the modeled organ and subsequent intensity-based registration of the lungs in oncological applications 1314 . Further improvement involves non-rigid transformation that allows to recover local deformation due to the shape changes in the lungs and should be considered as a potential extension of the model-based registration framework.

\section{References}

1. Maintz, J.B.A., Viergever, M.A.: A survey of medical image registration. MEDIA 2 (1998) 1-36

2. Wells, W.M., Viola, P., Atsumi, H., Nakajima, S., Kikinis, R.: Multi-modal volume regsitration by maximization of mutual information. MEDIA 1 (1996) 35-51

3. Rueckert, D., Sonoda, L.I., Hayes, C., Hill, D.L.G., Leach, M.O., Hawkes, D.J.: Non-rigid registration using free-form deformations: Application to breast $\mathrm{mr}$ images. IEEE TMI 18 (1999) 712-721

4. Besl, P.J., McKay, N.D.: A method for registration of 3d shapes. IEEE PAMI 14 (1992) 239-256

5. Rangarajan, A., Chui, H., Duncan, J.S.: Rigid point feature registration using mutual information. MEDIA 3 (1999) 425-440

6. Stewart, C.V., Lee, Y.L., Tsai, C.L.: An uncertainty-driven hybrid of intensitybased and feature-based registration with application to retinal and lung ct images. In: Proc. MICCAI. LNCS 3216 (2004) 870-877

7. Lelieveldt, B.P.F., Sonka, M., Bolinger, L., Scholz, T.D., van der Geest, H.K.R., Reiber, J.H.C.: Anatomical modeling with fuzzy implicit surface templates: Application to automated localization of the heart and lungs in thoracic mr volumes. CVIU 80 (2000) 1-20

8. Storti, D.W., Ganter, M.A., Nevrinceanu, C.: Tutorial on implicit solid modeling. Mathematica J 2 (1992) 70-78

9. Dempster, A.P., Laird, N.M., Rubin, D.B.: Maximum likelihood from incomplete data via the em algorithm. J Royal Stat Soc(B) 39 (1977) 1-38

10. Press, W.H., Flannery, B.P., Teukolsky, S.A., Vetterling, W.T.: Numerical Recipes in C: The Art of Scientific Computing. Cambridge Univ Press (1992) 
11. Ibáñez, L., Ng, L., Gee, J., Aylward, S.: Registration patterns: The generic framework for image registration of the insight toolkit. In: Proc. IEEE Int Symp Biomed Imaging. (2002) 345-348

12. McLeish, K., Hill, D.L.G., Atkinson, D.A., Blackall, J.M., Razavi, R.: A study of the motion and deformation of the heart due to respiration. IEEE TMI 21 (2002) $1142-1150$

13. Betke, M., Hong, H., Ko, J.P.: Automatic 3d registration of lung surfaces in computed tomography scans. In: Proc. MICCAI. LNCS 2208 (2001) 725-733

14. Coselmon, M.M., Balter, J.M., McShan, D.L., Kessler, M.L.: Mutual information based ct registration of the lung at exhale and inhale breathing states using thinplate splines. Med Phys 31 (2004) 2942-2948 